\author{
MASSIMILIANO AMARANTE and FABIO MACCHERONI
}

\title{
WHEN AN EVENT MAKES A DIFFERENCE
}

\begin{abstract}
For $(S, \Sigma)$ a measurable space, let $\mathcal{C}_{1}$ and $\mathcal{C}_{2}$ be convex, weak* closed sets of probability measures on $\Sigma$. We show that if $\mathcal{C}_{1} \cup$ $\mathcal{C}_{2}$ satisfies the Lyapunov property, then there exists a set $A \in \Sigma$ such that $\min _{\mu_{1} \in \mathcal{C}_{1}} \mu_{1}(A)>\max _{\mu_{2} \in \mathcal{C}_{2}} \mu_{2}(A)$. We give applications to Maxmin Expected Utility (MEU) and to the core of a lower probability.
\end{abstract}

KEY WORDS: Lyapunov theorem, Maximin expected utility, lower probability

\section{INTRODUCTION}

In the theory of decision making under uncertainty as well as in the theory of cooperative games, several questions can be reduced to the problem of whether or not two distinct sets of measures disagree on a set. For instance, if two Maxmin Expected Utility (MEU) preferences have the same utility on the prize space and the same willingness to bet, are they necessarily the same? Under which conditions, does the core of a lower probability coincide with the weak* closed and convex hull of any set of measures defining it? Both questions are answered in the affirmative if and only if one knows that there exists a set $A$ such that $\min _{\mu_{1} \in \mathcal{C}_{1}} \mu_{1}(A)>\max _{\mu_{2} \in \mathcal{C}_{2}} \mu_{2}(A)$, whenever $\mathcal{C}_{1}$ and $\mathcal{C}_{2}$ are two (convex, weak* closed) disjoint sets of measures. This is our main result, which we prove in the next section under the conditions stated therein. In Section 3, we provide a quick sample of the usefulness of Theorem 1, by answering the two questions stated above. We do not discuss, however, the full range of applications of Theorem 1. For another less immediate application, we refer the reader to Amarante and Filiz (2004), where our Theorem 1 turns out to be a key tool to characterize those events which 
are unambiguous either in the sense of Zhang (2002) or of Epstein and Zhang (2001). In general, we expect Theorem 1 to be widely applicable in areas different from the ones we consider such as in Quasi-Bayesian Statistics (due to the central role played by upper probabilities; see, for instance, Wasserman and Kadane (1990)) or in Social Choice Theory.

\section{MAIN RESULT}

If $\mu_{1}$ and $\mu_{2}$ are two probability measures on a $\sigma$-algebra $\Sigma$, then (by definition) $\mu_{1} \neq \mu_{2}$ means that there exists a set $A \in \Sigma$ such that $\mu_{1}(A)>\mu_{2}(A)$. Equivalently, the two disjoint sets $\left\{\mu_{1}\right\}$ and $\left\{\mu_{2}\right\}$ can be separated by means of a linear functional having an especially simple form, namely one that is defined by an indicator function. Here, we are concerned with extending this property to sets of measures which are not singletons.

Let $(S, \Sigma)$ be a measurable space and let $\Delta(\Sigma)$ denote the set of all (countably additive) probability measures on $\Sigma$. $\Delta(\Sigma)$ is a subset of the norm dual of the Banach space of bounded, $\Sigma$-measurable functions.

DEFINITION 1. Let $\mathcal{C}=\left\{\mu_{i}\right\}_{i \in I} \subset \Delta(\Sigma)$. We say that $\mathcal{C}$ has the Lyapunov property if the range of the vector measure $\left(\mu_{i}\right)_{i \in I}$ on $E$ is a convex and compact subset of $\mathbb{R}^{I}$ (equipped with the product topology), for all $E \in \Sigma$.

Notice that if $\mathcal{C}$ has the Lyapunov property and all of its elements are absolutely continuous with respect to a given probability measure, then any subset of $\mathcal{C}$ has the Lyapunov property, and that finite dimensional sets of nonatomic measures have this property (see Kingman and Roberston, 1968). Sets of measures with the Lyapunov property have special importance in the theory of decision making under uncertainty. For a decision maker described by a set of priors like in Gilboa and Schmeidler (1989) or in Ghirardato et al. (2003), the Lyapunov property corresponds to the demand that the class of unambiguous events in the sense of Nehring (1999) or Ghirardato et al. (2003) be "rich" (Section 3). 
THEOREM 1. Let $\mathcal{C}_{1}$ and $\mathcal{C}_{2}$ be convex, weak ${ }^{*}$ closed subsets of $\Delta(\Sigma)$ such that $\mathcal{C}_{1} \cup \mathcal{C}_{2}$ has the Lyapunov property. Then $\mathcal{C}_{1} \cap$ $\mathcal{C}_{2}=\emptyset$ if and only if there exists $A$ in $\Sigma$ such that

$$
\min _{\mu_{1} \in \mathcal{C}_{1}} \mu_{1}(A)>\max _{\mu_{2} \in \mathcal{C}_{2}} \mu_{2}(A)
$$

Proof. Since each $\mathcal{C}_{i}$ is weak* compact, then it is weak compact, and convexity of $\mathcal{C}_{i}$ implies that there exist a measure $\lambda_{i} \in \mathcal{C}_{i}$ such that $\mu_{i} \ll \lambda_{i}$ for all $\mu_{i} \in \mathcal{C}_{i}, i=1,2$ (see, for instance Chateaneuf et al., 2005). Hence, all the measures in $\mathcal{C}_{1} \cup \mathcal{C}_{2}$ are absolutely continuous with respect to $\lambda=\frac{1}{2} \lambda_{1}+\frac{1}{2} \lambda_{2}$, and the sets $\mathcal{C}_{1}^{\prime}$ and $\mathcal{C}_{2}^{\prime}$ of all Radon-Nikodym derivatives of elements of $\mathcal{C}_{1}$ and $\mathcal{C}_{2}$ are disjoint, weakly compact, and convex subsets of $\mathcal{L}^{1}(\lambda)$. The Separating Hyperplane Theorem (see, Dunford and Schwartz, 1954, V.2.10) guarantees that there exist $g_{0} \in \mathcal{L}^{\infty}(\lambda)-\{0\}$ such that

$$
\min _{f_{1} \in \mathcal{C}_{1}^{\prime}} \int g_{0} f_{1} \mathrm{~d} \lambda>\max _{f_{2} \in \mathcal{C}_{2}^{\prime}} \int g_{0} f_{2} \mathrm{~d} \lambda
$$

W.1.o.g. $0 \leqslant g_{0}(s) \leqslant 1$ for $\lambda$-almost all $s \in S$ (otherwise take $\left.\frac{g_{0}-\operatorname{essinf} g_{0}}{\| g_{0}-\text { essinf } g_{0} \|_{\infty}}\right)$.

By assumption, $\mathcal{C}_{1} \cup \mathcal{C}_{2}$ has the Lyapunov property. Hence, $\mathcal{C}_{1}^{\prime} \cup \mathcal{C}_{2}^{\prime}$ is thin in the sense of Kingman and Roberston (1968). By Lemma 1 in Kingman and Roberston (1968), $g_{0}=\chi_{A}+h$ where $A \in \Sigma$ and $h \in \mathcal{L}^{\infty}(\lambda)$ is such that $\int h f \mathrm{~d} \lambda=0$ for all $f \in$ $\mathcal{C}_{1}^{\prime} \cup \mathcal{C}_{2}^{\prime}$. For all $\mu \in \mathcal{C}_{1} \cup \mathcal{C}_{2}$, setting $f=\mathrm{d} \mu / \mathrm{d} \lambda$ we have

$$
\mu(A)=\int_{A} f \mathrm{~d} \lambda=\int \chi_{A} f \mathrm{~d} \lambda=\int\left(\chi_{A}+h\right) f \mathrm{~d} \lambda=\int g_{0} f \mathrm{~d} \lambda
$$

and Equation (1) becomes

$$
\min _{\mu_{1} \in \mathcal{C}_{1}} \mu_{1}(A)>\max _{\mu_{2} \in \mathcal{C}_{2}} \mu_{2}(A) .
$$

The converse is obvious.

COROLLARY 2. Under the assumptions of Theorem $1, \mathcal{C}_{1} \subseteq \mathcal{C}_{2}$ if and only if $\min _{\mu_{1} \in \mathcal{C}_{1}} \mu_{1}(A) \geqslant \max _{\mu_{2} \in \mathcal{C}_{2}} \mu_{2}(A)$ for all $A \in \Sigma$. 
Proof. Let $\min _{\mu_{1} \in \mathcal{C}_{1}} \mu_{1}(A) \geqslant \min _{\mu_{2} \in \mathcal{C}_{2}} \mu_{2}(A)$ for all $A \in \Sigma$. Assume that $\mathcal{C}_{1}$ is not contained in $\mathcal{C}_{2}$. Then there exists $\bar{\mu} \in$ $\mathcal{C}_{1} 1 \mathcal{C}_{2}$. Since $\mathcal{C}_{2} \cup\{\bar{\mu}\}$ is thin, Theorem 1 yields that there exists $B \in \Sigma$ such that $\bar{\mu}(B)<\mu_{2}(B)$ for all $\mu_{2} \in \mathcal{C}_{2}$. Therefore $\min _{\mu_{1} \in \mathcal{C}_{1}} \mu_{1} B \leqslant \bar{\mu}(B)<\min _{\mu_{2} \in \mathcal{C}_{2}} \mu_{2}(B)$, which is absurd. The converse is trivial.

We conclude this section, by proving another separation result. This extends an obvious property of two nonatomic measures: if $\mu_{1} \neq \mu_{2}$, there exist $A, B \in \Sigma, A \cap B=\emptyset$ such that $\mu_{1}(A)>\mu_{1}(B)$ and $\mu_{2}(A)<\mu_{2}(B)$. Notice that this is no longer true if the nonatomicity assumption is removed. In this form, the separation theorem turns out to be a basic tool in study unambiguous events in the sense of Zhang (2002) and Epstein and Zhang (2001) (see Amarante and Filiz, 2004).

COROLLARY 3. Under the assumptions in Theorem 1, there exist $A, B \in \Sigma, A \cap B=\emptyset$, such that $\mu_{1}(A)-\mu_{1}(B)>0>\mu_{2}(A)-$ $\mu_{2}(B)$ for any $\mu_{1} \in \mathcal{C}_{1}$ and any $\mu_{2} \in \mathcal{C}_{2}$.

Proof. Let $A \in \Sigma$ be such that $\mu_{1}(A)>\mu_{2}(A)$ for any $\mu_{i} \in$ $\mathcal{C}_{i}, i=1,2$. Since $\mathcal{C}_{1} \cup \mathcal{C}_{2}$ has the Lyapunov property, the range on $S$ of the vector measure defined by $\mathcal{C}_{1} \cup \mathcal{C}_{2}$ is compact and convex. Hence, for any $\alpha \in[0,1]$ there exists $B \in \Sigma$ such that $\mu_{1}(B)=\mu_{2}(B)=\alpha$ for all $\mu_{i} \in \mathcal{C}_{i}, \quad i=1,2$. Pick one such a $B$ so that $2 \mu(B)=\min _{\mu_{1} \in \mathcal{C}_{1}} \mu_{1}(A)+\max _{\mu_{2} \in \mathcal{C}_{2}} \mu_{2}(A)$ for all $\mu \in \mathcal{C}_{1} \cup$ $\mathcal{C}_{2}$. Then, $\mu_{1}(A)-\mu_{1}(B)>0$ and $\mu_{2}(A)-\mu_{2}(B)<0$ for all $\mu_{i} \in$ $\mathcal{C}_{i}, i=1,2$.

If $A \cap B \neq \emptyset$, write $B=(A \cap B) \cup B^{\prime}$ and $A=(A \cap B) \cup A^{\prime}$. Then, for any $\mu_{i} \in \mathcal{C}_{i}, \quad i=1,2$,

$$
\begin{aligned}
\mu_{1}(A)-\mu_{1}(B) & =\mu_{1}(A \cap B)+\mu_{1}\left(A^{\prime}\right)-\mu_{1}(A \cap B)-\mu_{1}\left(B^{\prime}\right) \\
& =\mu_{1}\left(A^{\prime}\right)-\mu_{1}\left(B^{\prime}\right) \\
\mu_{2}(A)-\mu_{2}(B) & =\mu_{2}\left(A^{\prime}\right)-\mu_{2}\left(B^{\prime}\right)
\end{aligned}
$$

and $A^{\prime}$ and $B^{\prime}$ do the job. 


\section{APPLICATION: MEU PREFERENCES AND LOWER PROBABILITIES}

In the theory of decision making under uncertainty, one is concerned with a decision maker ranking the elements of a set $\mathcal{A}$ of mappings $a: S \rightarrow X$, where $S$ is the state space and $X$ the prize space. For the sake of simplicity, let $X$ be a convex subset of a vector space and $\mathcal{A}$ be the set of all simple and measurable functions from $S$ to $X$. The decision maker's ranking $\succeq$, is said to satisfy the MEU criterion if and only if for $a, b \in \mathcal{A}$

$$
a \succeq b \Leftrightarrow \min _{\mu \in \mathcal{C}} \int(u \circ a) \mathrm{d} \mu \geqslant \min _{\mu \in \mathcal{C}} \int(u \circ b) \mathrm{d} \mu,
$$

where $u: X \rightarrow \mathbb{R}$ is a nonconstant and affine utility function on the prize space, and $\mathcal{C}$ is a weak* closed and convex set of finitely additive probability measures on $(S, \Sigma)$. The willingness to bet of a MEU decision maker is the lower probability

$$
\rho(A)=\min _{\min \in \mathcal{C}} \mu(A), \quad \forall A \in \Sigma .
$$

The core of a lower probability $\rho$ is the set core $(\rho)$ of all finitely additive probability measures $v$ on $(S, \Sigma)$ such that $v \geqslant \rho$.

Preferences satisfying the MEU criterion have been axiomatized in Gilboa and Schmeidler (1989). In Marinacci (2002) and Chateaneuf et al. (2005) necessary and sufficient conditions on $\succeq$ are given that guarantee that all the measures in $\mathcal{C}$ be countably additive. An event $A \in \Sigma$ is unambiguous in the sense of Nehring (1999) or Ghirardato et al. (2003) if $\mu(A)=\mu^{\prime}(A)$ for all $\mu, \mu^{\prime} \in \mathcal{C}$. In Amarante (2005) (Proposition 4), it was shown that (i) the class of unambiguous events is "rich", that is there exist unambiguous events of measure $\alpha$ for every $\alpha \in[0,1]$, and (ii) there exists a countably additive, nonatomic probability measure on the class of unambiguous events if and only if $\mathcal{C}$ has the Lyapunov Property.

In the context of MEU, a natural question is whether or not two MEU preferences with the same utility on the prize space and the same willingness to bet are necessarily the same 
preference. A related question in the theory of lower probabilities is whether or not the weak* closed and convex set $\mathcal{C}$ defining a lower probability $\rho$ coincides with its core. The following example, due to Huber and Strassen (1973), answers negatively to both questions.

EXAMPLE 1. Let $S=\{1,2,3\}, X=\mathbb{R}, \mu=\left(\frac{1}{2}, \frac{1}{2}, 0\right), v=\left(\frac{4}{6}, \frac{1}{6}, \frac{1}{6}\right)$. Consider two MEU preferences, $\succeq_{1}$ and $\succeq_{2}$, with $u_{1}(x)=$ $u_{2}(x)=x$ for any $x \in \mathbb{R}$ and sets of priors

$$
\mathcal{C}_{1}=c_{0}\{\mu, v\} \quad \text { and } \quad \mathcal{C}_{2}=\left\{\left(\frac{3+t}{6}, \frac{3-t-s}{6}, \frac{s}{6}\right): 0 \leqslant s, t \leqslant 1\right\} .
$$

It is readily checked that:

- $\rho(A)=\min _{\mu_{1} \in \mathcal{C}_{1}} \mu_{1}(A)=\min _{\mu_{2} \in \mathcal{C}_{2}} \mu_{2}(A)=\rho_{2}(A)$ for all $A \subset S$, but $\succeq_{1}$ is different from $\succeq_{2}$;

- $\mathcal{C}_{1}$ is a weak* closed and convex set defining the lower probability $\rho_{1}$, and it is strictly included in core $\left(\rho_{1}\right)$ (which coincides with $\mathcal{C}_{2}$ ).

Both conclusions are reverted under the assumptions of Theorem 1 as the next two corollaries show. In reading Corollary 4 , notice that point 1 . amounts to say that $\succeq_{1}$ is more ambiguity averse than $\succeq_{2}$ (see Ghirardato and Marinacci, 2002), and remember that $x A y$ is the mapping from $S$ to $X$ taking value $x$ on $A$ and $y$ on $A^{c}$.

COROLLARY 4. Let $\succeq_{1}$ and $\succeq_{2}$ be two $M E U$ preferences with (weak* closed and convex) sets of priors $\mathcal{C}_{1}$ and $\mathcal{C}_{2}$ contained in $\Delta(\Sigma)$ and such that $\mathcal{C}_{1} \cup \mathcal{C}_{2}$ has the Lyapunov property. Then the following conditions are equivalent:

(1) For all $a \in \mathcal{A}$ and $z \in X$,

$$
a \succeq_{1} z \Rightarrow a \succeq_{2} z \text {. }
$$

(2) For all $x, y, z \in X$ such that $x \succeq_{i} y$ for $i=1,2$, and all $A \in \Sigma$,

$$
x A y \succeq_{1} z \Rightarrow x A y \succeq_{2} z
$$

(3) $u_{1}$ is a positive affine transformation of $u_{2}$ and $\rho_{1} \leqslant \rho_{2}$.

In particular, if $u_{1}=u_{2}$ and $\rho_{1}=\rho_{2}$, then $\succeq_{1}$ coincides with $\succeq_{2}$. 
COROLLARY 5. Let $\rho$ be a lower probability such that core $(\rho) \subset \Delta(\Sigma)$ and core $(\rho)$ has the Lyapunov property. Then core $(\rho)$ is the weak ${ }^{*}$ closed and convex hull of any subset $\mathcal{K}$ of $\Delta(\Sigma)$ such that

$$
\rho(A)=\inf _{v \in \mathcal{K}} v(A), \quad \forall A \in \Sigma
$$

The easy proofs are omitted.

\section{ACKNOWLEDGEMENT}

We thank Mohammed Abdellaoui (the Editor) and an anonymous referee for helpful suggestions. The financial support of the Ministero dell'Istruzione, dell'Università e della Ricerca is gratefully acknowledged.

\section{REFERENCES}

Amarante, M. (2005), Ambiguity, measurability and multiple priors, Economic Theory 26, 995-1006.

Amarante, M. and Filiz, E. (2004), Ambiguous Events and Maxmin Expected Utility, Columbia University discussion paper \# 0405-09.

Chateaneuf, A., Maccheroni, F., Marinacci, M. and Tallon, J.-M. (2005), Monotone continuous multiple priors, Economic Theory 26, 973-982.

Dunford, N. and Schwartz, J.T. (1954). Linear Operators, Part I: General Theory. Wiley-Interscience, London.

Epstein, L. G. and Zhang, J. (2001), Subjective probabilities on subjectively unambiguous events, Econometrica 69, 265-306.

Ghirardato, P. and Marinacci, M. (2002), Ambiguity made precise: a comparative foundation, Journal of Economic Theory 102, 251-289.

Ghirardato, P., Maccheroni, F. and Marinacci, M. (2003), Differentiating ambiguity and ambiguity attitude, Journal of Economic Theory 118, 133173.

Gilboa, I. and Schmeidler, D. (1989), Maxmin expected utility with a nonunique prior, Journal of Mathematical Economics 18, 141-153.

Huber, P. J. and Strassen, V. (1973), Minimax tests and the NeymanPearson lemma for capacities, Annals of Statistics 1, 251-263.

Kingman, J. F. C. and Robertson, A.P. (1968), On a Theorem of Lyapunov, Journal of the London Mathematical Society 43, 347-351. 
Marinacci, M. (2002), Probabilistic sophistication and multiple priors, Econometrica 70, 755-764.

Nehring, K. (1999), Capacities and probabilistic beliefs: A precarious coexistence, Mathematical Social Sciences 38, 197-213.

Wasserman, L. A. and Kadane, J. B. (1990), Bayes' theorem for Choquet capacities, Annals of Statistics 18, 1328-1339.

Zhang, J. (2002), Subjective ambiguity, expected utility and choquet expected utility, Economic Theory 20, 159-181.

Addresses for correspondence: Massimiliano Amarante, Department of Economics, Columbia University, NY, USA.

Fabio Maccheroni, Istituto di Metodi Quantitativi and IGIER, Università Bocconi, Milan, Italy. E-mail: fabio.maccheroni@uni-bocconi.it 